\title{
RADIATION REACTION AND RENORMALIZATION VIA CONSERVATION LAWS OF THE POINCARÉ GROUP
}

\author{
Yu. Yaremko \\ Institute for Condensed Matter Physics of NASU, \\ 1 Svientsitskii St., UA-79011 Lviv, Ukraine
}

(Received June 17, 2003; received in final form June 15, 2004)

\begin{abstract}
We consider the self-action problem in classical electrodynamics of a point-like charge arbitrarily moving in flat space-time of four or six dimensions. A consistent regularization procedure is proposed which exploits the symmetry properties of the theory. The energy-momentum and angular momentum balance equations allow us to derive the radiation reaction forces in both 4D and $6 \mathrm{D}$. It is shown that a point-like source in $6 \mathrm{D}$ possesses an internal angular momentum with the magnitude which is proportional to the square of acceleration. $6 \mathrm{D}$ action functional contains, apart from the usual "bare" mass, an additional renormalization constant which corresponds to the curvature of the world line (i. e. to the magnitude of internal angular momentum of "bare" particle). It is demonstrated that the Poincaré-invariant six-dimensional electrodynamics is renormalizable theory.
\end{abstract}

Key words: classical electrodynamics, higher dimensions, radiation reaction, Poincaré group, conservation laws.

PACS number(s): 03.50.De, 11.10.Gh

\section{INTRODUCTION}

Recently [1,2], there has been a considerable interest in the renormalization procedure in classical electrodynamics of a point particle moving in flat space-time of arbitrary dimensions. The main task is to derive the analogue of the well-known Lorentz-Dirac equation [3]. Following the scheme proposed by Dirac in his classical paper [3], Gal'tsov in [1] decomposes vector potential of a point-like charge $A_{\text {ret }}^{\mu}=A_{\mathrm{S}}^{\mu}+A_{\mathrm{R}}^{\mu}$. The first term, $A_{\mathrm{S}}^{\mu}$, is the half-sum of the retarded, $A_{\text {ret }}^{\mu}$, and the advanced, $A_{\text {adv }}^{\mu}$, solutions of the D'Alembert equation with a pointlike source. Since $A_{\mathrm{S}}^{\mu}$ is singular in the immediate vicinity of the world line, the subscript "S" stands for "singular" as well as "symmetric". Because $A_{\mathrm{S}}^{\mu}$ is just singular as $A_{\text {ret }}^{\mu}$, removing it from the retarded solution gives the potential $A_{\mathrm{R}}^{\mu}=1 / 2\left(A_{\mathrm{ret}}^{\mu}-A_{\mathrm{adv}}^{\mu}\right)$ that well behaves near the world line. Since $A_{\mathrm{R}}^{\mu}$ satisfies the homogeneous wave equation, it can be interpreted as a free radiation field. Hence the subscript "R" stands for "regular" as well as "radiative".

In classical electrodynamics of a point-like charge arbitrarily moving in flat space-time of four dimensions the singular part gives a divergent self-energy while the regular one leads to standard radiation reaction force. The unphysical "bare" mass involved in the action integral absorbs the divergent self-energy of a point charge within the renormalization procedure and then becomes the observable finite rest mass of the particle $[1,3,4]$. In six dimensions the Coulomb potential of a charge scales as $|\mathbf{r}|^{-3}[1,5]$. Inevitable infinities arising in higherdimensional electrodynamics are stronger than in four dimensions. For this reason Gal'tsov [1] claims, that "in even dimensions higher than four, divergences cannot be removed by the mass renormalization".

To make classical electrodynamics in six dimensions a renormalizable theory, in [6] the six-dimensional analogue of the relativistic particle with rigidity [7-9] is substituted for the structureless point charge whose Lagrangian is proportional to worldline length. New Lagrangian involves, apart from usual "bare mass", an additional regularization constant which absorbs one extra divergent term. In [2] the procedure of regularization in any dimensions is elaborated by using functional analysis. To get a renormalizable theory for the even dimension $D>4$, the original Lagrangian for free relativistic particle with "bare" mass is modified by introducing $D / 2-2$ extra (higher derivative) terms.

So, in six dimensions the whole renormalization procedure involves two arbitrary constants. Since there are three types of divergences in six dimensions, Gal'tsov doubts whether "all divergences arising in higherdimensional electrodynamics can be absorbed in this way" [1]. The introduction of higher derivatives in the particle action term seems for him not reasonable enough since it "drastically changes the initial theory".

Contrary to $[1,2]$ where the authors deal with equations of motion, Kosyakov [6] calculates the flux of energy-momentum and derive the radiation-reaction force by adding appropriate Schott term. In the present paper we consider also the conserved quantities corresponding to the invariance of the theory under proper homogeneous Lorentz transformations. They give an additional information which clarifies the essence of renormalization procedure. In such a way we reformulate the problem of renormalizability within the problem of Poincaré invariance of a closed particle plus field system. The conservation laws are an immovable fulcrum about which tips the balance of truth regarding renormalization and radiation reaction. Either nonrenormalizable theory or renormalizable one should be compatible with the Poincaré symmetry. 


\section{PRELIMINARIES}

The standard variational principle is formulated $[1,2,6]$ for a composite system of point-like charged particle and its own electromagnetic field:

$$
I_{\text {total }}=I_{\text {part }}+I_{\text {int }}+I_{\text {field }}
$$

Here

$$
\begin{aligned}
& I_{\text {field }}=-\frac{1}{4 \Omega_{D-2}} \int d^{D} y F^{\mu \nu} F_{\mu \nu} \\
& I_{\text {part }}=-m \int d \tau \sqrt{-\dot{z}^{2}}
\end{aligned}
$$

and the interaction term given by

$$
I_{\mathrm{int}}=e \int d \tau A_{\mu} \dot{z}^{\mu}
$$

The particle's world line $\zeta: \mathbb{R} \rightarrow \mathbb{M}_{D}$ is described by the functions $z^{\alpha}(\tau)$ which give the particle's coordinates as functions of proper time $\tau ; \dot{z}^{\alpha}(\tau)=d z^{\alpha}(\tau) / d \tau$. By $\Omega_{D-2}$ we denote the area of a $(D-2)$-dimensional sphere of unit radius:

$$
\Omega_{D-2}=2 \frac{\pi^{(D-1) / 2}}{\Gamma\left(\frac{D-1}{2}\right)} .
$$

The action (1) is invariant under infinitesimal transformation (translations and rotations) which constitute the Poincaré group. According to Noether's theorem, these symmetry properties can be used for the derivation of conservation laws, i. e. those quantities that do not change with time.

Strictly speaking, the action integral (1) may be used to derive trajectories of the test particles, when the field is given a priori. It may also be used to derive $D$ dimensional Maxwell equations, if the particle trajectories are given a priori. Simultaneous variation with respect to both field and particle variables is incompatible since the Lorentz force will always be ill defined in the immediate vicinity of the particle's world line.

Our consideration is founded on field (2) and interaction (3) terms of the Eq. (1). They constitute the action functional which governs the propagation of electromagnetic field produced by a moving charge (i.e., the Maxwell equations with point-like source):

$$
\square A^{\alpha}(y)=-\Omega_{D-2} j^{\alpha}(y) .
$$

The Liénard-Wiechert fields are the solutions of the Maxwell equations with point-like sources. One can substitute these fields in the conservation laws to rewrite them in terms of particle variables.

The components of energy-momentum carried by the electromagnetic field are $[4,6,10]$

$$
p_{\mathrm{em}}^{\nu}(\tau)=P \int_{\Sigma} d \sigma_{\mu} T^{\mu \nu}
$$

where $d \sigma_{\mu}$ is the vectorial surface element on an arbitrary space-like hypersurface $\Sigma$. The components of the electromagnetic field's stress-energy tensor

$$
\Omega_{D-2} T^{\mu \nu}=F^{\mu \lambda} F_{\lambda}^{\nu}-1 / 4 \eta^{\mu \nu} F^{\kappa \lambda} F_{\kappa \lambda}
$$

have a singularity on the particle trajectory. In Eq.(6) capital letter $P$ denotes the principal value of the singular integral, defined by removing from $\Sigma$ an $\varepsilon$-sphere around the particle and then passing to the limit $\varepsilon \rightarrow 0$.

The angular momentum tensor of the electromagnetic field is written as $[10,11]$

$$
M_{\mathrm{em}}^{\mu \nu}(\tau)=P \int_{\Sigma} d \sigma_{\alpha}\left(y^{\mu} T^{\alpha \nu}-y^{\nu} T^{\alpha \mu}\right)
$$

Conservation of the space part $M_{\mathrm{em}}^{i j}$ of the tensor $M_{\mathrm{em}}^{\mu \nu}$ is due to invariance under space rotations. Conservation of the mixed space-time components, $M_{\mathrm{em}}^{0 i}$, takes place due to invariance under Lorentz transformations.

\section{COORDINATE SYSTEM}

Using the retarded Green function [1, Eq. (3.4)] associated with the D'Alembert operator $\square$ and the chargecurrent density vector $e \int d \tau u^{\alpha}(u) \delta(y-z(u))$ we construct the retarded Liénard-Wiechert potential in even dimensions:

$$
A_{\mu}=\left(\frac{1}{2 \pi} \frac{1}{r} \frac{d}{d u}\right)^{(D-4) / 2} e \frac{u_{\mu}(u)}{r} .
$$

An appropriate coordinate system for flat space-time is very important for the volume integration (6) and (8). We calculate how much electromagnetic field momentum and angular momentum flow across a world tube of constant radius $r$ enclosing the world line $\zeta$ (Bhabha tube [12], see Fig. 1). A world tube is a disjoint union of (retarded) spheres of constant radii $r$ centered on a world line $\zeta: \mathbb{R} \rightarrow \mathbb{M}_{D}$ of the particle. The sphere $S(z(u), r)$ is the intersection of the future light cone, generated by null rays emanating from $z(u) \in \zeta$ in all possible directions,

$$
\begin{aligned}
C(z(u)) & =\left\{y \in \mathbb{M}_{D}:\left(y^{0}-z^{0}(u)\right)^{2}\right. \\
& \left.=\sum_{i}\left(y^{i}-z^{i}(u)\right)^{2}, y^{0}-z^{0}(u)>0\right\}
\end{aligned}
$$

and tilted hyperplane $\Sigma(z(u), r)$ : 
$\Sigma(z(u), r)=\left\{y \in \mathbb{M}_{6}: u_{\alpha}(u)\left(y^{\alpha}-z^{\alpha}(u)-u^{\alpha}(u) r\right)=0\right\}$.

The points on the sphere are distinguished by spherical polar angles involved in the space components $n^{i^{\prime}}$ of the null vector $n^{\alpha^{\prime}}=\left(1, n^{i^{\prime}}\right)$, namely $(\cos \phi \sin \vartheta, \sin \phi \sin \vartheta, \cos \vartheta)$ in four dimensions, or $\quad\left(\cos \varphi \sin \vartheta_{1} \sin \vartheta_{2} \sin \vartheta_{3}, \sin \varphi \sin \vartheta_{1} \sin \vartheta_{2} \sin \vartheta_{3}\right.$, $\left.\cos \vartheta_{1} \sin \vartheta_{2} \sin \vartheta_{3}, \quad \cos \vartheta_{2} \sin \vartheta_{3}, \cos \vartheta_{3}\right)$ in six dimensions.

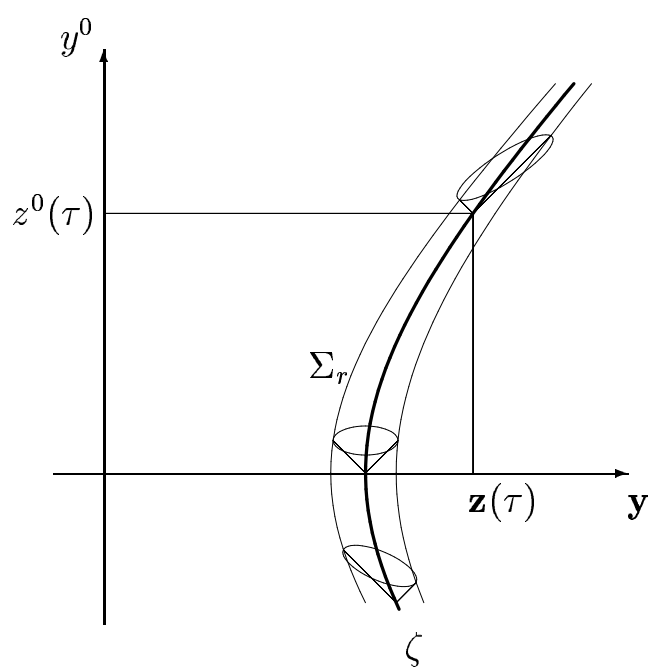

Fig. 1. Integration region considered in the evaluation of the bound and emitted conserved quantities produced by all points of the world line up to the end point with coordinates $\left(z^{0}(\tau), \mathbf{z}(\tau)\right)$. Retarded spheres $\left.\left.S(z(u), r), u \in\right]-\infty, \tau\right]$, of constant radii $r$ constitute a thin world tube $\Sigma_{r}$ enclosing the world line $\zeta$. The sphere $S(z(u), r)$ is the intersection of the future light cone with vertex at the point $z^{\mu}(u) \in \zeta$, and shifted hyperplane $\Sigma(z(u), r)$ which is orthogonal to the particle's velocity $u^{\mu}(u)$.

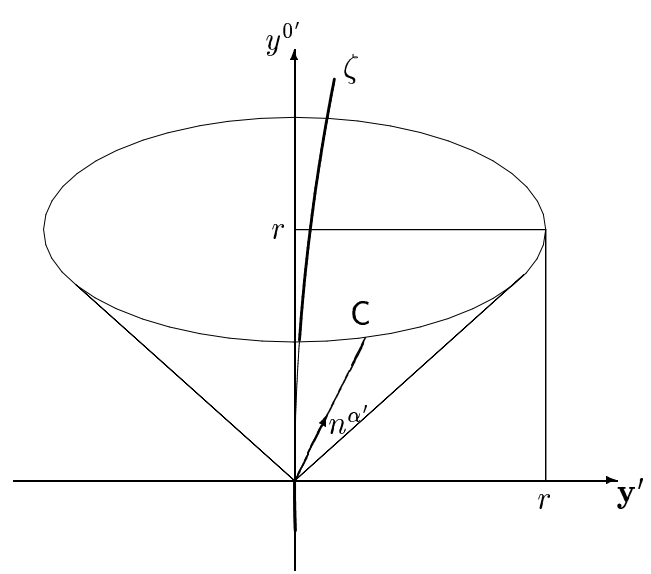

Fig. 2. In MCLF the retarded distance is the distance between any point on the spherical light front $S(0, r)=\left\{y \in \mathbb{M}_{6}:\left(y^{0^{\prime}}\right)^{2}=\sum_{i}\left(y^{i^{\prime}}\right)^{2}, y^{0^{\prime}}=r>0\right\}$ and the particle. The charge is placed in the coordinate origin; it is momentarily at rest. The point $\mathrm{C} \in S(0, r)$ is linked to the coordinate origin by a null ray characterized by the angles $\vartheta^{A}$ specifying its direction on the cone. The vector with components $n^{\alpha^{\prime}}$ is tangent to this null ray.
To understand the situation more thoroughly, we pass to particle's momentarily comoving Lorentz frame (MCLF) where the particle is momentarily at rest at the retarded instant $u$ (see Fig. 2). The charge is placed in the coordinate origin; the sphere $S(0, r)$ is the intersection of the future light cone with vertex at the origin and hyperplane $y^{0^{\prime}}=r$.

As usual, we call a retarded distance the distance $r$ between any point on the spherical light front $S(0, r)$ and the particle, taken in MCLF. In the laboratory frame the points on this sphere have the following coordinates:

$$
\begin{aligned}
y^{\alpha} & =z^{\alpha}(u)+r \Lambda_{\alpha^{\prime}}^{\alpha}(u) n^{\alpha^{\prime}} \\
& =z^{\alpha}(u)+r k^{\alpha} .
\end{aligned}
$$

The flat space-time $\mathbb{M}_{D}$ becomes a disjoint union of world tubes $\Sigma_{r}, r>0$, enclosing the particle trajectory.

\section{RENORMALIZATION AND RADIATION REACTION IN FOUR DIMENSIONS}

It is straightforward to substitute the components $F_{\alpha \beta}=\partial_{\alpha} A_{\beta}-\partial_{\beta} A_{\alpha}$ into Eq. (7) to calculate the electromagnetic field's stress-energy tensor. Direct calculations show $[4,11]$ that either energy-momentum (6) or angular momentum (8) carried by the retarded Liénard-Wiechert field contains two quite different parts: (i) the bound part which is permanently "attached" to the charge and is carried along with it; (ii) the radiation part detaches itself from the charge and leads an independent existence. The former is divergent while the latter is finite. The bound parts depend on the state of particle's motion at the vicinity of the observation time only while the radiative parts are accumulated with time (see Fig. 3). Within the regularization procedure the bound terms are coupled with energy-momentum and angular momentum of "bare" sources, so that already renormalized characteristics $G_{\text {part }}^{\alpha}$ of charged particles are proclaimed to be finite. Noether quantities which are properly conserved become:

$$
G^{\alpha}=G_{\mathrm{part}}^{\alpha}+G_{\mathrm{rad}}^{\alpha}
$$

On rearrangement, the total four-momentum of our composite particle plus field system looks as follows:

$$
P^{\mu}=p_{\text {part }}^{\mu}+\frac{2}{3} e^{2} \int_{-\infty}^{\tau} d u\left(a^{\lambda} a_{\lambda}\right) u^{\mu}(u),
$$

where $a^{\lambda}(u)=d u^{\lambda} / d u$ is $\lambda$-th component of the particle acceleration. Similarly, the total angular momentum is

$$
M^{\mu \nu}=z^{\mu}(\tau) p_{\text {part }}^{\nu}-z^{\nu}(\tau) p_{\text {part }}^{\mu}
$$




$$
\begin{aligned}
& +\frac{2}{3} e^{2} \int_{-\infty}^{\tau} d u(a \cdot a)\left[z^{\mu}(u) u^{\nu}(u)-z^{\nu}(u) u^{\mu}(u)\right] \\
& +\frac{2}{3} e^{2} \int_{-\infty}^{\tau} d u\left[u^{\mu}(u) a^{\nu}(u)-u^{\nu}(u) a^{\mu}(u)\right]
\end{aligned}
$$

where the dot denotes a scalar product. The particle fourmomentum $p_{\text {part }}$ is already renormalized.

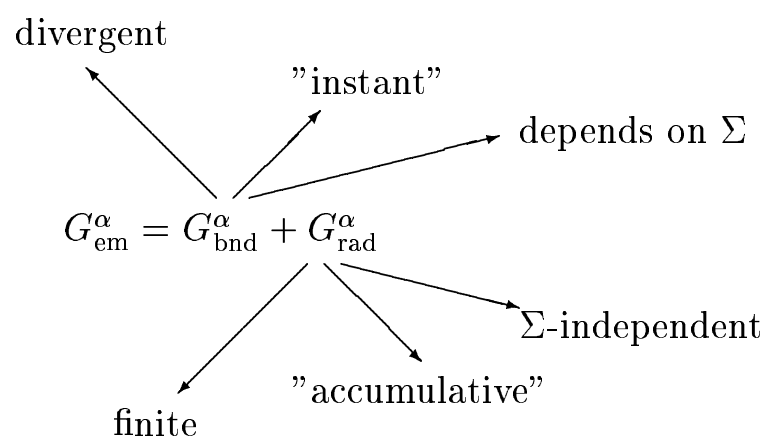

Fig. 3. The bound term $G_{\mathrm{bnd}}^{\alpha}$ and the radiative term $G_{\mathrm{rad}}^{\alpha}$ constitute Noether quantity $G_{\mathrm{em}}^{\alpha}$ carried by the electromagnetic field. The former diverges while the latter is finite. The bound component depends on instant characteristics of charged particles while the radiative one is accumulated with time. The form of the bound term heavily depends on choosing an integration surface $\Sigma$ while the radiative term does not depend on $\Sigma$.

Time differentiation of these conserved quantities gives the system of ten equations in four variables $p_{\text {part }}^{\mu}$ and their time derivatives [13]. Having differentiated (15) and taking into account the differential consequence of Eq. (14)

$$
\dot{p}_{\text {part }}^{\mu}(\tau)=-\frac{2}{3} e^{2}(a \cdot a) u^{\mu}(\tau)
$$

we arrive at the equality which explains how the fourmomentum of charged particle depends on its velocity and acceleration:

$$
u \wedge p_{\text {part }}=-\frac{2}{3} e^{2} u \wedge a
$$

(The symbol $\wedge$ denotes the wedge product.) Hence the particle four-momentum contains, apart from the usual velocity term, also a contribution from the acceleration when the particle is charged:

$$
p_{\text {part }}^{\mu}=m u^{\mu}-\frac{2}{3} e^{2} a^{\mu}
$$

Since $(u \cdot a)=0$, the scalar product of particle fourvelocity on the first-order time-derivative of particle fourmomentum (16) is as follows:

$$
\left(\dot{p}_{\text {part }} \cdot u\right)=\frac{2}{3} e^{2}(a \cdot a)
$$

Similarly, the scalar product of particle acceleration on the particle four-momentum (18) is given by

$$
\left(p_{\text {part }} \cdot a\right)=-\frac{2}{3} e^{2}(a \cdot a)
$$

Summing up (19) and (20) we obtain

$$
\frac{d}{d \tau}\left(p_{\mathrm{part}} \cdot u\right)=0
$$

Therefore, $m$ is already renormalized rest mass of the particle [5]. We are sure that the well-known Teitelboim's expression (18) for the four-momentum of pointlike charged particle [4] as well as the Lorentz-Dirac equation [3] arise from the total energy-momentum and angular momentum balance equations.

\section{RADIATION REACTION IN SIX DIMENSIONS}

Taking $D=6$ in (9), one has again that LiénardWiechert potential in six dimensions depends on particle acceleration:

$$
A_{\mu}=\frac{e}{2 \pi}\left[\frac{a_{\mu}(u)}{r^{2}}+\frac{u_{\mu}(u)}{r^{3}}\left(1+r a_{k}\right)\right] .
$$

Here $a_{k}=a_{\alpha} k^{\alpha}$ is the component of the particle acceleration in the direction of $k^{\alpha}:=\Lambda_{\alpha^{\prime}}(u) n^{\alpha^{\prime}}$.

The electromagnetic field is defined in terms of this potential by $F_{\alpha \beta}=A_{\beta, \alpha}-A_{\alpha, \beta}$. Having used the differentiation rule $[6$, eqs. $(2),(3)]$

$$
\frac{\partial u}{\partial y^{\mu}}=-k_{\mu}, \quad \frac{\partial r}{\partial y^{\mu}}=-u_{\mu}+\left(1+r a_{k}\right) k_{\mu}
$$

we obtain

$$
F=\frac{e}{2 \pi}\left(\frac{u \wedge a}{r^{3}}+V \wedge k\right)
$$

where

$$
\begin{aligned}
V_{\mu} & =\frac{3 u_{\mu}}{r^{4}}+\frac{3\left(a_{\mu}+2 u_{\mu} a_{k}\right)}{r^{3}} \\
& +\frac{\dot{a}_{\mu}+u_{\mu} \dot{a}_{k}+3 a_{\mu} a_{k}+3 u_{\mu} a_{k}^{2}}{r^{2}} .
\end{aligned}
$$

The overdot means the derivative with respect to retarded time $u$. The Liénard-Wiechert field (24) coincides with the field obtained in $[6$, Eq. (14)] where the "mostly minus" metric signature should be replaced by the 
"mostly plus" one and the overall coefficient $e / 2 \pi$ should be substituted for 1 (or for $1 / 3$ in Eq. (15) of the E-print version corrected by the author).

It is straightforward to substitute the components (24) into Eq. (7) to calculate the electromagnetic field's stressenergy tensor. Following [6], we present $T^{\alpha \beta}$ as a sum of radiative and bound components,

$$
T^{\alpha \beta}=T_{\mathrm{rad}}^{\alpha \beta}+T_{\mathrm{bnd}}^{\alpha \beta}
$$

The radiative part scales as $r^{-4}$ :

$$
\frac{8 \pi^{2}}{3} T_{\mathrm{rad}}^{\alpha \beta}=\frac{e^{2}}{4 \pi^{2}} \frac{k^{\alpha} k^{\beta}}{r^{4}} V_{(-2)}^{\mu} V_{\mu}^{(-2)}
$$

where the components $V_{\mu}^{(-2)}$ of six-vector $V_{(-2)}$ is defined by Eq. (25). The others $T_{(-\kappa)}$ constitute the bound part of the Maxwell energy-momentum tensor density:

$$
T_{\mathrm{bnd}}^{\alpha \beta}=T_{(-8)}+T_{(-7)}+T_{(-6)}+T_{(-5)}
$$

(Each term has been labelled according to its dependence on the distance $r$.)

According to [6], the outward-directed surface element $d \sigma_{\mu}$ of a five-cylinder $r=$ const in $\mathbb{M}_{6}$ is

$$
d \sigma_{\mu}=\left[-u_{\mu}+\left(1+r a_{k}\right) k_{\mu}\right] r^{4} d \Omega_{4} d u
$$

where $d \Omega_{4}=d \vartheta_{1} d \vartheta_{2} d \vartheta_{3} d \phi \sin \vartheta_{1} \sin ^{2} \vartheta_{2} \sin ^{3} \vartheta_{3}$ is the element of solid angle in five dimensions. The angular integration can be handled via the relations

$$
\begin{aligned}
& \int d \Omega_{4}=\frac{8 \pi^{2}}{3} \\
& \int d \Omega_{4} n^{\alpha} n^{\beta}=\frac{8 \pi^{2}}{15}\left(\eta^{\alpha \beta}+u^{\alpha} u^{\beta}\right) \\
& \int d \Omega_{4} n^{\alpha} n^{\beta} n^{\gamma} n^{\kappa}=\frac{8 \pi^{2}}{105}\left[\left(\eta^{\alpha \beta}+u^{\alpha} u^{\beta}\right)\left(\eta^{\gamma \kappa}+u^{\gamma} u^{\kappa}\right)\right. \\
& +\left(\eta^{\alpha \gamma}+u^{\alpha} u^{\gamma}\right)\left(\eta^{\beta \kappa}+u^{\beta} u^{\kappa}\right) \\
& \left.+\left(\eta^{\alpha \kappa}+u^{\alpha} u^{\kappa}\right)\left(\eta^{\beta \gamma}+u^{\beta} u^{\gamma}\right)\right]
\end{aligned}
$$

The integral of the polynomial in odd powers of $n^{\alpha}:=$ $k^{\alpha}-u^{\alpha}$ vanishes.

We are now concerned with the integration of (6). The volume integration of the bound part of the stress-energy tensor over the world tube $\Sigma_{r}$ of constant radius $r$ reveals that the bound energy-momentum is a function of the end points only:

$p_{\text {bnd }}^{\mu}=\frac{e^{2}}{4 \pi^{2}}\left[\frac{3}{2} \frac{u^{\mu}(u)}{r^{3}}+\frac{12}{5} \frac{a^{\mu}(u)}{r^{2}}+2 \frac{(a \cdot a) u^{\mu}(u)}{r}\right]_{u \rightarrow-\infty}^{u=\tau}$
(The matter is that the total (retarded) time derivatives arise from angular integration.) If the charged particle is asymptotically free at the remote past, we obtain the Coulomb-like self-energy of constant value. The upper limit drastically depends on the value of $r$. To evaluate the bound part of six-momentum in the neighborhood of the particle we take the limit $r \rightarrow 0$. If $r$ tends to zero, $p_{\text {bnd }}^{\mu} \rightarrow \infty$. The divergences are absorbed by the individual particle's six-momentum within the renormalization procedure based on the Noether quantities (see Fig. 3 and Eq. (13)). The total energy-momentum of a closed system of an arbitrarily moving charge and its electromagnetic field is equal to the sum

$$
P^{\mu}=p_{\text {part }}^{\mu}+p_{\text {rad }}^{\mu}
$$

where $p_{\text {rad }}$ is the radiative part of electromagnetic field's energy-momentum which does not depend on $r$ at all:

$$
\begin{aligned}
p_{\text {rad }}^{\mu} & =\frac{e^{2}}{4 \pi^{2}} \int_{-\infty}^{\tau} d u\left(\frac{4}{5}(\dot{a} \cdot \dot{a}) u^{\mu}-\frac{6}{35}(a \cdot a) \dot{a}^{\mu}\right. \\
& \left.+\frac{3}{7} a^{\mu}(a \cdot a)^{\cdot}+2(a \cdot a)^{2} u^{\mu}\right)
\end{aligned}
$$

(We denote $(a \cdot a)^{\cdot}$ the derivative $d(a \cdot a) / d u$.)

Volume integration of the angular momentum tensor density shows that the bound angular momentum depends on the state of the particle's motion at the observation instant only:

$M_{\mathrm{bnd}}^{\mu \nu}=\frac{e^{2}}{4 \pi^{2}} \lim _{r \rightarrow 0}\left(z^{\mu} P_{\mathrm{bnd}}^{\nu}-z^{\nu} P_{\mathrm{bnd}}^{\mu}+\frac{12}{5} \frac{u^{\mu} a^{\nu}-u^{\nu} a^{\mu}}{r}\right)$.

By symbol $P_{\text {bnd }}$ we mean the expression in between the squared brackets of Eq. (31).

It is worth noting that $M_{\mathrm{bnd}}^{\mu \nu}$ contains, apart from the usual term of the type $z \wedge p_{\text {part }}$, also an extra term which can be interpreted as the "shadow" of internal angular momentum. It prompts that the bare "core" possesses a "spin".

Total angular momentum of our composite particle plus the field system is written as

$$
M^{\mu \nu}=M_{\mathrm{part}}^{\mu \nu}+M_{\mathrm{rad}}^{\mu \nu}
$$

where $M_{\text {rad }}$ is the radiative part (36) of the electromagnetic field's angular momentum which depends on all the previous motion of the source:

$$
M_{\mathrm{rad}}^{\mu \nu}=\frac{e^{2}}{4 \pi^{2}}\left\{\int_{-\infty}^{\tau} d u\left(z^{\mu} P_{\mathrm{rad}}^{\nu}-z^{\nu} P_{\mathrm{rad}}^{\mu}\right)\right.
$$




\section{YU. YAREMKO}

$$
\begin{aligned}
& +\int_{-\infty}^{\tau} d u\left[\frac{4}{5}\left(a^{\mu} \dot{a}^{\nu}-a^{\nu} \dot{a}^{\mu}\right)\right. \\
& \left.\left.+\frac{64}{35}(a \cdot a)\left(u^{\mu} a^{\nu}-u^{\nu} a^{\mu}\right)\right]\right\} .
\end{aligned}
$$

Here $P_{\text {rad }}$ denotes the integrand of Eq. (33).

With (34) in mind we assume that already renormalized angular momentum tensor of the particle has the form

$$
M_{\text {part }}^{\mu \nu}=z^{\mu} p_{\text {part }}^{\nu}-z^{\nu} p_{\text {part }}^{\mu}+u^{\mu} \pi_{\text {part }}^{\nu}-u^{\nu} \pi_{\text {part }}^{\mu}
$$

In [7-9] the extra momentum $\pi_{\text {part }}$ is due to additional degrees of freedom associated with the acceleration involved in the Lagrangian function for the rigid particle.

Our next task is to derive expressions which explain how six-momentum and angular momentum of the charged particle depend on its velocity and acceleration. Having performed the time differentiation of Eq. (32) we obtain the following energy-momentum balance equation:

$$
\begin{aligned}
\dot{p}_{\text {part }}^{\mu} & =-\frac{e^{2}}{4 \pi^{2}}\left(\frac{4}{5}(\dot{a} \cdot \dot{a}) u^{\mu}-\frac{6}{35}(a \cdot a) \dot{a}^{\mu}\right. \\
& \left.+\frac{3}{7} a^{\mu}(a \cdot a)^{\cdot}+2(a \cdot a)^{2} u^{\mu}\right) .
\end{aligned}
$$

(All the particle characteristics are evaluated at the instant of observation $\tau$.) Having differentiated (35) and taking into account (38) we arrive at the equality which does not contain $\dot{p}_{\text {part }}$ :

$$
\begin{aligned}
& u \wedge\left(p_{\text {part }}+\dot{\pi}_{\text {part }}+\frac{e^{2}}{4 \pi^{2}} \frac{64}{35}(a \cdot a) a\right) \\
& +a \wedge\left[\pi_{\text {part }}+\frac{e^{2}}{4 \pi^{2}} \frac{4}{5} \dot{a}\right]=0 .
\end{aligned}
$$

A scrupulous analysis of the consistency of the energymomentum balance equation (38) and angular momentum balance equation (39) reveals that the sixmomentum of charged particle contains two (already renormalized) constants [14, Appendix]:

$$
\begin{aligned}
& p_{\text {part }}^{\beta}=m u^{\beta}+\mu\left(-\dot{a}^{\beta}+\frac{3}{2}(a \cdot a) u^{\beta}\right) \\
& +\frac{e^{2}}{4 \pi^{2}}\left[\frac{4}{5} \ddot{a}^{\beta}-\frac{8}{5} u^{\beta}(a \cdot a)^{\cdot}-\frac{64}{35}(a \cdot a) a^{\beta}\right] .
\end{aligned}
$$

The first one, $m$, looks as a rest mass of the charge. But the true rest mass is identical to the scalar product of the six-momentum and six-velocity [5]. Since the scalar product depends on the square of acceleration as well as its time derivative

$$
m_{0}=-\left(p_{\text {part }} \cdot u\right)=m+\frac{\mu}{2}(a \cdot a)-\frac{e^{2}}{4 \pi^{2}} \frac{2}{5}(a \cdot a)
$$

the renormalization constant $m$ is formal parameter and its physical sense is not clear.

The second, $\mu$, is intimately connected with the internal angular momentum $s_{\text {part }}:=u \wedge \pi_{\text {part }}$ of the particle:

$$
s_{\mathrm{part}}^{\alpha \beta}=\mu\left(u^{\alpha} a^{\beta}-u^{\beta} a^{\alpha}\right)-\frac{e^{2}}{4 \pi^{2}} \frac{4}{5}\left(u^{\alpha} \dot{a}^{\beta}-u^{\beta} \dot{a}^{\alpha}\right) .
$$

But the magnitude of $s_{\text {part }}$ is not constant:

$$
\begin{aligned}
s^{2} & =-\frac{1}{2} s_{\alpha \beta}^{\mathrm{part}} s_{\mathrm{part}}^{\alpha \beta}=\mu^{2}(a \cdot a)+\mu \frac{e^{2}}{5 \pi^{2}}(a \cdot a)^{.} \\
& +\frac{e^{4}}{25 \pi^{4}}\left((\dot{a} \cdot \dot{a})+(a \cdot a)^{2}\right) .
\end{aligned}
$$

Therefore, this name cannot be understood literally.

Having substituted the right-hand side of Eq. (40) for the particle's six-momentum in the energy-momentum balance equation (38), we derive the Lorentz-Dirac equation of motion of a charged particle under the influence of its own electromagnetic field. An external device adds covariant external force $F_{\text {ext }}$ to the right-hand side of this expression.

Expression (40) was originally obtained by Kosyakov in [6, Eq. (37)] (see Eq. (38) in E-print version corrected by the author). The derivation is based upon the consideration of energy-momentum conservation only. The author constructs an appropriate Schott term to ensure the orthogonality of the radiation reaction force to the particle six-velocity. The formula define the bound sixmomentum of a point-like charged particle which is involved in the energy-momentum balance equation (see Eq. (40) in E-print version of the paper and Eq. (32) of the present paper where this momentum is denoted as $\left.p_{\text {part }}\right)$. The bound momentum differs from the one of the particle being "dressed" in electromagnetic "fur" (see Eq. (42) in E-print version of [6]). The latter contains also a contribution from the radiated energy-momentum (33) carried by the electromagnetic field of an accelerated particle. The six-momentum of "dressed" particle is involved in six-dimensional analogue of relativistic generalization of Newton's second law [6, Eq. (38)] where the loss of energy due to radiation is taken into account.

We face the problem if two renormalization constants, $m$ and $\mu$, are sufficient to absorb the three divergences of our divergent bound six-momentum (31). The structure of this expression heavily depends on the integration surface. Having integrated the electromagnetic field's stressenergy tensor (7) over hyperplane $\Sigma_{t}=\left\{y \in \mathbb{M}_{6}: y^{0}=\right.$ $t\}$, we obtain 


$$
\begin{aligned}
p_{\mathrm{bnd}}^{\mu} & =\int_{\Sigma_{t}} d \sigma_{0} T_{\mathrm{bnd}}^{0 \mu} \\
& =\frac{e^{2}}{4 \pi^{2}}\left[\frac{3}{35} \frac{-12 u^{0} u^{\mu}+40\left(u^{0}\right)^{3} u^{\mu}+\eta^{0 \mu}\left(-3 / 2+12\left(u^{0}\right)^{2}\right)}{(t-u)^{3}}\right. \\
& +\frac{3}{35} \frac{-5 a^{\mu}+31 a^{0} u^{0} u^{\mu}+33 a^{\mu}\left(u^{0}\right)^{2}+3 \eta^{0 \mu} a^{0}}{(t-u)^{2}} \\
& \left.+\frac{1}{35} \frac{37 a^{0} a^{\mu}+71(a \cdot a) u^{0} u^{\mu}+\eta^{0 \mu}(a \cdot a)}{t-u}\right]_{u \rightarrow-\infty}^{u \rightarrow t} .
\end{aligned}
$$

The lower limit is equal to zero while the upper one tends to infinity. The coefficients are quite different from that in Eq. (31) while the number of divergences is still equal to three.

Further we choose the tilted hyperplane $\sigma_{\tau}=\{y \in$ $\left.\mathbb{M}_{6}: u_{\mu}(\tau)\left(y^{\mu}-z^{\mu}(\tau)\right)=0\right\}$ which is intimately connected with the momentarily co-moving Lorentz frame of the charge at the observation instant $\tau$ (cf. $r$-shifted hyperplane (11)). In MCLF the particle is momentarily at rest at the time $\tau$. To apply our previous result we make such a Lorentz transformation $\Omega$ that a tilted hyperplane $\sigma_{\tau}$ becomes $\Sigma_{t^{\prime}}=\left\{y \in \mathbb{M}_{6}: y^{0^{\prime}}=t^{\prime}\right\}$ :

$$
\begin{aligned}
p_{\mathrm{bnd}}^{\mu} & =\int_{\sigma_{t}} d \sigma_{\nu} T_{\mathrm{bnd}}^{\nu \mu} \\
& =\int_{y^{0^{\prime}}=t^{\prime}} d \sigma_{0^{\prime}} T_{\mathrm{bnd}}^{0^{\prime} \alpha^{\prime}} \Omega_{\alpha^{\prime}} \mu \\
& =\Omega^{\mu}{ }_{\alpha^{\prime}} p_{\mathrm{bnd}}^{\alpha^{\prime}} .
\end{aligned}
$$

It is now a straightforward (but tedious) matter to calculate the components of bound six-momentum in MCLF. They contains two divergences only:

$$
\begin{aligned}
& p_{\mathrm{bnd}}^{0^{\prime}}=\frac{e^{2}}{4 \pi^{2}} \lim _{u^{\prime} \rightarrow t^{\prime}}\left[\frac{3}{2} \frac{1}{\left(t^{\prime}-u^{\prime}\right)^{3}}+2 \frac{(a \cdot a)}{t^{\prime}-u^{\prime}}\right], \\
& p_{\mathrm{bnd}}^{i^{\prime}}=\frac{e^{2}}{4 \pi^{2}} \lim _{u^{\prime} \rightarrow t^{\prime}}\left[-\frac{6}{5} \frac{\dot{a}^{i^{\prime}}}{t^{\prime}-u^{\prime}}\right] .
\end{aligned}
$$

Since the structure of bound six-momentum is changeable, it would make no sense to disrupt the bonds between different powers of the small parameter $r$ in (31). It is sufficient to assume that a charged particle possesses its own (already renormalized) six-momentum $p_{\text {part }}$ which is transformed as a usual six-vector under the Poincaré group. A careful analysis of the energymomentum and angular momentum balance equations reveals how $p_{\text {part }}$ depends on six-velocity, six-acceleration etc. In $\mathbb{M}_{4}$ the solution (18) contains one renormalization parameter: the rest mass $m$. If we deal with six- dimensional flat space-time than two renormalization parameters, $m$ and $\mu$, arise (see Eq. (40)).

\section{CONCLUSIONS}

We examine whether the renormalizabolity is a necessary condition for consistency of the local field theory with fundamental principles such as energy-momentum conservation and the conservation of the total angular momentum. A careful analysis with the use of regularization procedure compatible with the Poincaré symmetry shows, that the usual particle part of the initial action integral (1) which is proportional to the worldline lenght is inconsistent with $I_{\text {field }}$ and $I_{\text {int }}$ in six dimensions. Indeed, the angular momentum tensor of a structureless particle

$$
M_{\text {part }}^{\mu \nu}=z^{\mu} p_{\text {part }}^{\nu}-z^{\nu} p_{\text {part }}^{\mu}
$$

corresponds to $I_{\text {part }}$ given by (2). Having analyzed the angular momentum balance equations one has again

$$
u \wedge\left(p_{\text {part }}+\frac{e^{2}}{4 \pi^{2}} \frac{64}{35}(a \cdot a) a\right)+\frac{e^{2}}{4 \pi^{2}} \frac{4}{5} a \wedge \dot{a}=0
$$

instead of (39). Its solution is a motion with constant velocity where $p_{\text {part }}^{\mu}$ do not change. Hence the action functional based on the higher-derivative Lagrangian for a "rigid" relativistic particle $[7,8]$ should be substituted for $I_{\text {part }}$ in (2). It involves two renormalization constants $[2,6]$; it is sufficient to renormalize all the divergences arising in six-dimensional electrodynamics.

Nonrenormalizable theory contradicts the differential consequences of the conserved quantities which arise from the invariance of the system under space rotations and Lorentz transformations.

Volume integration of the energy-momentum and angular momentum carried by the electromagnetic field shows that a charged particle is supplemented with the bound electromagnetic "cloud" which has its own (divergent) momentum and angular momentum. Correspond- 
ing characteristics of the "bare" charge absorb them within the regularization procedure based on Noether conservation laws (see Figure 3). The radiative parts of the electromagnetic field's energy-momentum and angular momentum detach the charge and lead an independent existence. They are involved in energy-momentum and angular momentum balance equations which determine how already renormalized particle's momentum and angular momentum depend on its velocity, acceleration etc (see Eq. (18) for $\mathbb{M}_{4}$ and Eq. (40) for $\mathbb{M}_{6}$ ).

It is worth noting that in six dimensions a test particle (i. e., point charge which itself does not influence the field) is the rigid particle. Its momentum is given by expression (40) where $e^{2}$ tends to zero; it is not parallel to six-velocity. The problem of motion of such particles in the external electromagnetic field is considered in [15].

\section{ACKNOWLEGEMENT}

The author would like to thank Professor B. P. Kosyakov, Professor V. Tretyak, and Dr. A. Duviryak for helpful discussions and critical comments.
[1] D. V. Gal'tsov, Phys. Rev. D 66, 025016 (2002).

[2] P. O. Kazinski, S. L. Lyakhovich, A. A. Sharapov, Phys. Rev. D 66, 025017 (2002).

[3] P. A. M. Dirac, Proc. R. Soc. A 167, 148 (1938).

[4] C. Teitelboim, Phys. Rev. D 1, 1572 (1970).

[5] B. P. Kosyakov, Fiz. Elem. Chast. i Atom. Yadra 34, 1564 (2003) (in Russian) [Phys. Part. Nucl. 34, 808 (2003)]; e-print hep-th/0208035 (2002).

[6] B. P. Kosyakov, Teor. Mat. Fiz. 119, 119 (1999) (in Russian) [Theor. Math. Phys. 199, 493 (1999)]; e-print hepth/0207217 (2002).

[7] M. S. Plyushchay, Phys. Lett. B 253, 50 (1991); 235, 47
(1990); Int. J. Mod. Phys. A 4, 3851 (1989); Mod. Phys. Lett. A 3, 1299 (1988).

[8] M. Pavšič, Phys. Lett. B 221, 264 (1989).

[9] V. V. Nesterenko, J. Math. Phys. 32, 3315 (1991).

[10] F. Rohrlich, Classical Charged Particles (Redwood City, CA: Addison-Wesley, 1990).

[11] C. A. López, D. Villarroel, Phys. Rev. D 11, 2724 (1975).

[12] H. J. Bhabha, Proc. R. Soc. A 172, 384 (1939).

[13] Yu. Yaremko, J. Phys. A 35, 831 (2002); 35, 9441 (2002); 36, 5149 (2003); 36, 5159 (2003).

[14] Yu. Yaremko, J. Phys. A 37, 1079 ( 2004).

[15] V. V. Nesterenko, Int. J. Mod. Phys. 6A, 3989 (1991).

\title{
ЗБЕРЕЖУВАНІ ВЕЛИЧИНИ ГРУПИ ПУАНКАРЕ: ПЕРЕНОРМУВАННЯ ТА РЕАКЦІЯ ВИПРОМІНЮВАННЯ
}

\author{
Ю. Яремко \\ Інститут фізики конденсованих систем НАН України \\ вул. Свєнціцького, 1, Лъвів, 79011, Україна
}

\begin{abstract}
Досліджено проблему самодії у класичній електродинаміці точкового заряду, що довільно рухається в чотиривимірному та шестивимірному просторах Мінковського. Запропоновано процедуру перенормування, узгоджену із симетрійними властивостями теорії. Із законів збереження енергії-імпульсу та моменту кількости руху знайдено сили реакції випромінювання в обох, чотиривимірному та шестивимірному, випадках. Виявилось, що точковий заряд у шестивимірній електродинаміці має внутрішній кутовий момент, амплітуда якого пропорційна до квадрата прискорення частинки. Відповідний функціонал дії містить, окрім звичної “затравочної” маси, ще й додаткову константу перенормування, пов'язану з кривизною світової лінії (тобто $з$ амплітудою внутрішнього кутового моменту "голої" частинки). Показано, що Пуанкаре-інваріянтна шестивимірна електродинаміка є перенормовною теорією.
\end{abstract}

